\title{
Annie Benveniste (ed). Se faire violence. Analyse des coulisses de la recherche
}

\section{Sylvie Ayimpam}

\section{(2) OpenEdition}

\section{Journals}

Édition électronique

URL : http://journals.openedition.org/anthropodev/311

DOI : 10.4000/anthropodev.311

ISSN : 2553-1719

Éditeur

APAD - Association pour l'anthropologie du changement social et du développement

Édition imprimée

Date de publication : 1 septembre 2014

Pagination : 163-166

ISBN : 9791093476018

ISSN : 2276-2019

Référence électronique

Sylvie Ayimpam, «Annie Benveniste (ed). Se faire violence. Analyse des coulisses de la recherche », Anthropologie \& développement [En ligne], 40-41 | 2014, mis en ligne le 01 septembre 2016, consulté le 24 septembre 2020. URL : http://journals.openedition.org/anthropodev/311 ; DOI : https://doi.org/ 10.4000/anthropodev.311

La revue Anthropologie \& développement est mise à disposition selon les termes de la Licence Creative Commons Attribution 4.0 International. 


\section{Se faire violence. Analyse des coulisses de la recherche}

Annie Benveniste (ed), Paris, Téraèdre, 2013, 189 p.

Sylvie Ayimpam

Les auteurs de cet ouvrage collectif nous livrent ici un aspect du terrain de recherche généralement peu abordé : l'expérience de la violence telle que vécue pendant l'enquête. Bien qu'ils aient travaillé sur des terrains géographiquement et thématiquement différents, on est frappé par la similitude des expériences de la violence qu'ils rapportent. L'atelier qui a précédé la rédaction de l'ouvrage a donné l'occasion aux auteurs de revenir d'abord dans la discussion orale sur les modes d'objectivation de la violence dans leur articulation avec les conditions de production de la recherche. Ensuite, il leur a permis d'aborder autrement le rapport du chercheur à la violence en montrant comment cette dernière devenait un analyseur des matériaux, des objets analysés et des affects. D'ailleurs du point de vue des auteurs, cette subjectivé des chercheurs n'est pas réduite à la réflexivité. Bien qu'ils se soient également préoccupés d'interroger leurs places et postures en tant que chercheurs, ils sont allés beaucoup plus loin, en s'obligeant à revenir sur les effets - parfois occultés - du surgissement de la violence au cours de l'enquête ou dans le retour des " affects » ressentis sur le terrain. Ils ont ainsi pu passer d'une violence objectivée et perçue sur le terrain comme violence des populations, des conflits, des travestissements de l'histoire, à la violence faite au chercheur (p.23).

Le travail ethnographique d'Annie Benveniste à Soweto s'est fait dans le cadre d'un programme de recherche portant sur le " rôle de l'école dans la transformation des rapports sociaux » en Afrique du Sud (p.158). Son travail avait pour objectif de collecter les témoignages afin d'analyser le poids du passé dans la transmission, et de comprendre comment se tissaient les relations intergénérationnelles dans la concurrence entre l'histoire officielle et la revendication de groupes ou de personnes singulières à faire entendre leur histoire. D'ailleurs cette histoire officielle, celle de la libération, celle de la désagrégation, ne cadrait pas vraiment avec les réalités observées sur le terrain à Soweto (p.167). En interrogeant les habitants de Soweto sur leur passé pour comprendre la manière dont se faisait la transmission, la chercheure n'a obtenu dans le discours de ses interlocuteurs qu'une comparaison avec la dépossession du présent plaidant ainsi en faveur du passé : perte du respect, perte du rapport aux ainés, perte de valeurs, perte d'autorité vis-à-vis des enfants, perte de savoirs domestiques, etc. Mais si le rapport douloureux au passé a d'abord été escamoté au profit d'une plainte concernant le présent, il est vite revenu au travers des discours sur les souffrances du corps qui parlent de l'oppression de l'apartheid (p.175). Les discours apparaissaient ainsi comme signes du refoulement des violences passées, refoulement dévoilé par les souffrances du 
présent. La chercheure se retrouve confrontée ainsi à travers les récits de ses enquêtés aux dires de la violence : la violence du chômage et de ses conséquences sur l'insécurité psychique et physique des résidents du quartier liée en bonne partie aux violences interfamiliales ; la violence représentée par la menace de la criminalité vénale dans un environnement où les habitants ne possèdent pas grand chose ; la violence de l'épidémie de sida, et par dessus tout les violences intrafamiliales (p.182). Alors qu'elle était partie sur le terrain avec pour objectif d'étudier la manière dont la transmission institutionnelle ou familiale du passé de l'apartheid et des luttes accompagnait le changement politique, la chercheure s'est retrouvée prise dans les effets de la violence exercée par la souffrance de ses interlocuteurs sur le terrain (p.186).

La mise à l'épreuve de l'enquêteur par la souffrance rencontrée sur le terrain se retrouve aussi dans la contribution d'Aude Rabaud, à propos d'une enquête menée dans les quartiers populaires de la région de Bordeaux. Durant cette enquête où elle a fait une longue immersion sur le terrain, elle s'est retrouvée prise à partie dans les conflits de voisinage, tout comme elle a été aussi témoin de drames divers (p.32). En choisissant d'emménager dans ce quartier où elle allait vivre pendant près de cinq ans, le terrain l'a malgré elle appelée à se focaliser sur le caractère structurant d'un certain registre de violence dans ce milieu (p.37). Etant témoin des conduites intempestives, répétitives, tapageuses, elle a préféré faire abstraction du stress qu'elle développait face aux nuisances extérieures se conjuguant, en refusant de considérer son environnement immédiat comme agressif. En effet, face à ce "stress résidentiel très marqué ", elle ne s'est pas autorisée à prendre au sérieux le désordre de ses émotions et l'impact des affects dans le rapport au terrain. En revenant après plus d'une dizaine d'années sur le sentiment permanent de peur qu'elle a ressenti pendant ces années de terrain, elle souligne combien les sentiments de dette et de culpabilité restent encore vifs et inchangés. Si la violence physique ne lui faisait pas peur, la chercheure était en revanche tétanisée par l'ampleur des rencontres et des échanges quotidiens. Craignant que des voisins ne frappent pour la énième fois à la porte, et usant de stratagèmes parfois ridicules pour faire croire qu'elle n'était pas là, elle espérait se sortir de cette manière de cette pression (pp.72-73). A l'occasion de cet exercice réflexif, elle s'est rendue compte de l'actualité de l'emprise des émotions ressenties pendant l'enquête. D'ailleurs, la fin des échanges avec ses enquêtés fut trop rapide, car elle a disparu du quartier et mis fin aux rencontres de manière trop brutale, avec un arrière-goût d'inachevé. Mais ce sont surtout les relations interpersonnelles allant au-delà de l'enquête qui ont pesé, à son avis, sur la dimension affective des conditions de la recherche. Elle se demande alors si la confiance entre elle et les interlocuteurs avec lesquels elle avait sympathisé était réelle ou si elle n'était qu'un paravent au senti- 
ment ambigu de flouer les personnes avec lesquelles elle ne faisait que voisiner (p.73).

De son coté, Giacomo Loperfido discute de la notion de "terrain difficile » qui renvoie dans son cas à l'image externe du milieu fasciste, milieu qui est fermé envers des " étrangers » et d'hostilité affichée envers le monde antifasciste auquel appartient pourtant le chercheur. La difficulté de l'enquête tenait aussi au fait que le milieu universitaire antifasciste ne tendait pas à produire des analyses affranchies de préjugés idéologiques sur le néofascisme. D'où l'ambiguïté de la démarche de l'ethnographe qui n'échappe d'ailleurs pas aux informateurs, qui peuvent alors y suspecter la mauvaise foi de l'ennemi (p.80). Pour le chercheur dont l'antifascisme est un a priori fondateur, l'objectif est de parvenir à séparer démarche méthodologique d'une part, et subjectivité, valeurs, construction idéologique de la critique d'autre part, notions qui se superposent pourtant dans le travail d'enquête, et qui rendent l'analyse plus complexe pour le chercheur (p.98). L'ethnographe devient ainsi le point de contact entre deux discours contradictoires qui supposent chacun l'exclusion de l'autre, avec une fragilité structurelle liée à sa position entre deux systèmes de classification antagonistes (p.99), avec une construction diabolique de l'autre assez violente dans les deux milieux, sous-tendue par une logique de défense contre le danger d'infiltration et de contagion (p.101). Aussi l'ethnographe qui traverse la frontière interne entre deux champs de l'identité et de l'altérité crée, par sa présence, un espace de proximité pouvant mettre fin au système de ségrégation entre deux groupes, tout en libérant l'autre de sa fonction de représenter ce que le groupe n'est pas. Le chercheur montre que ce processus de construction de l'identité peut entrer en crise, et cette crise peut déclencher des comportements de défense et d'agressivité vis-à-vis du chercheur (p.114). Mais le défi pour le chercheur ici fût de brouiller la vision bien ordonnée des choses, une représentation idéalisée des réalités où les valeurs étaient bien distinctes, entre celles que partageait le chercheur où les valeurs étaient bien, et celles que prônaient les autres qui représentaient le mal (p.115). Il a pris le risque de franchir la frontière de l'altérité et d'écouter le récit des autres, chose qui lui apprit beaucoup sur luimême et sur la société où il avait été formé (p.116).

Partie sur le terrain sans planifier de faire une enquête anthropologique, mais plutôt pour tenter de comprendre ce qui s'était passé en 2008 lors de la flambée xénophobe qui avait touché tout un ensemble de townships autour de Johannesburg en Afrique du Sud, Judith Hayem s'est retrouvée dans une position clairement sortie de son rôle de chercheure, en s'engageant comme militante improvisée de l'association African Diaspora Forum (ADF), une association de défense des communautés étrangères en Afrique du Sud (p.120). Le malaise qu'a ressenti la chercheure lors de ces évènements est moins lié, selon elle, à la violence qu'elle a vécu qu'au fait d'être confrontée à la réalité xénophobe de l'Etat sud-africain post- 
apartheid, à sa brutalité à l'égard des étrangers, au relent de ses méthodes autoritaires, à ses propos ouvertement racistes et à la passivité de nombreux sudafricains, même des anciens militants de la lutte anti-apartheid. Lors de cet épisode sur le terrain, la chercheure témoigne avoir été personnellement soumise à cette violence aux plans psychologique et physique. Et à l'occasion de cette expérience elle eût beaucoup de difficultés à se situer face à son terrain et à ses convictions personnelles. Pour arriver à poursuivre ses recherches sur le terrain, il lui a fallu d'une part prendre acte de sa déception face à ce qui s'était passé sur son terrain de recherche, interroger son sentiment latent d'avoir été flouée, et d'autre part dépasser le traumatisme et la blessure personnelle face à la violence dont elle fût le témoin et qu'elle avait elle-même ressentie (p.121). Cette expérience l'a amenée à analyser son rapport au terrain de recherche sud africain dans lequel elle travaillait depuis de nombreuses années, et à faire cet exercice réflexif sur deux plans. D'abord sur l'expérience de la violence qu'elle a vécue en tant que militante sortie de son rôle de chercheure, violence dont la paradoxale capacité de cristallisateur a été plus révélatrice que la qualification de la violence; ensuite sur l'analyse des séquences politiques et la difficulté du chercheur à gérer affect et concept quand la politique à l'œuvre sur son terrain de recherche suscite en lui un profond désarroi après l'avoir enthousiasmé (p.122).

A travers la lecture de cet ouvrage, on voit qu'une expérience pénible de l'enquête de terrain, où le chercheur " rencontre » la violence sous une forme ou une autre, où il vit un certain désarroi, un certain sentiment de trahison du terrain, une certaine tristesse, etc., demande pour être réappropriée, que le chercheur effectue d'abord un " détour " puis un " retour ». Cela traverse tous les cas exposés dans cet ouvrage. Après le vécu de la violence sur le terrain, le temps semble un facteur important au chercheur pour ré-apprivoiser le terrain ou ré-apprivoiser le rapport au terrain, sans passion fatale ou sans rejet brutal (p.154). L'ouvrage apparaît ainsi comme un long voyage-retour sur les différents terrains des auteurs, et le résultat, en termes de déconstruction de la " naturalisation " de la violence, n'en est qu'édifiant. Les auteurs ont ainsi réussi à rendre compte, avec une distance qu'on ne peut qu'apprécier, de l'évolution de leur propre rapport à la violence de leur terrain de recherche. Cet ouvrage ne réunit que quatre textes : on aurait sans doute aimé avoir un peu plus d'expériences de ce genre rapportées. Mais en même temps, le fait qu'il y ait eu très peu de textes a sans doute laissé plus d'espace à chaque auteur, bien plus qu'il n'en aurait eu dans un ouvrage plus volumineux, pour revenir en détails et en profondeur sur sa propre expérience et son ressenti de la violence sur son terrain de recherche. 